\title{
Comparative Study between RIPASA and Modified Alvarado Scoring System in the Diagnosis of Acute Appendicitis
}

\author{
Shivakumar T. ${ }^{1}$, Tilak Ramu G² , Savitha Rani B. B. ${ }^{3}$ \\ 1, 2 Department of General Surgery, Sri Siddhartha Medical College and Research \\ Institute, Tumkur, Karnataka, India. ${ }^{3}$ Department of Community Medicine, Sri \\ Siddhartha Medical College and Research Institute, Tumkur, Karnataka, India.
}

\section{ABSTRACT}

\section{BACKGROUND}

Acute appendicitis is one of the common surgical emergencies. Acute appendicitis can be converted to complicated appendicitis such as gangrenous appendicitis or perforated appendix, which is associated with greater mortality and morbidity. Therefore, surgeons have been forced to operate the patient when the diagnosis is in question. The accuracy of the clinical examination varies greatly depending on the experience of the examiner. Surgeons have traditionally accepted negative findings of appendectomy and the removal of a normal appendix. There are many scoring systems developed such as Alvarado and Modified Alvarado scoring system. RIPASA, a new scoring system has been developed to help in the diagnosis of acute appendicitis in Asian countries. In our study, we have compared the Modified Alvarado and RIPASA scoring system for their sensitivity, specificity and diagnostic accuracy.

\section{METHODS}

This observational study was done in Sri Siddhartha Medical College and Research Institute between October 2018 and March 2020 among 70 patients with clinical diagnosis of acute appendicitis and undergoing appendectomy, after obtaining their consent. Both Modified Alvarado and RIPASA score were calculated for all the patients. The operative findings and postoperative histopathology report were compared with the two scoring systems. Sensitivity, specificity, positive predictive value, negative predictive value and diagnostic accuracy were calculated.

\section{RESULTS}

At optimal cut-off point of 7.5 for RIPASA, the sensitivity and specificity were $94.1 \%$ and $33.3 \%$ respectively. At the cut-off threshold of 7.0 for modified Alvarado score, the sensitivity and specificity is $30.9 \%$ and $6.0 \%$ respectively. The diagnostic accuracy of RIPASA was $63.31 \%$ which is better than Modified Alvarado score.

\section{CONCLUSIONS}

Both Modified Alvarado and RIPASA score have significant differences in sensitivity, specificity and diagnostic accuracy in the diagnosis of acute appendicitis. In the present study RIPASA score was found to be more accurate in detecting cases of acute appendicitis when compared to the Modified Alvarado scoring system. Use of scoring systems in developing countries would be useful in detecting acute appendicitis quickly and in further preventing progression of disease and its complication.

\section{KEY WORDS}

Acute Appendicitis, RIPASA Score, Modified Alvarado Scoring System, Histopathology, Sensitivity, Specificity and Diagnostic Accuracy
Corresponding Author: Dr. Shivakumar T., Professor, Department of General Surgery, Sri Siddhartha Medical College and Research Institute, Tumkur, Karnataka, India.

E-mail: dr_shivakumar123@rediffmail.com

DOI: $10.14260 / \mathrm{jemds} / 2021 / 62$

How to Cite This Article:

Shivakumar T, Ramu TG, Rani SBB, et al. Comparative study between RIPASA and modified alvarado scoring system in the diagnosis of acute appendicitis. J Evolution Med Dent Sci 2021;10(05):279-283, DOI: $10.14260 / \mathrm{jemds} / 2021 / 62$

Submission 22-09-2020,

Peer Review 01-12-2020,

Acceptance 07-12-2020,

Published 01-02-2021.

Copyright (C) 2021 Shivakumar T. et al. This is an open access article distributed under Creative Commons Attribution License [Attribution 4.0 International (CC BY 4.0)] 


\section{BACKGROUND}

Acute appendicitis is one of the most common surgical emergencies in clinical practice, with an estimated lifetime prevalence of approximately 1 in 7 . it is one of the common causes of acute abdomen and emergency abdominal surgery. ${ }^{1}$ The appendicitis is commonly diagnosed using the patient's signs, symptoms and the symptoms of appendicitis can often correlate with various other disorders, especially in children, adults, females of reproductive age group and elderly. ${ }^{2}$ In patients whom the diagnosis cannot be made based on the patient's complaints and examination, close follow up, radiological and lab investigations can be helpful. The most commonly used radiological investigation are ultrasound and computed tomography scan which has shown to be more specific than ultrasound in diagnosing acute appendicitis. ${ }^{3}$ But elevated price and low accessibility are some factors that prevents its use specially in developing countries. ${ }^{4}$ In 1886 Reginald Heber Fitz described the classical signs and symptoms of acute appendicitis as a disease entity. ${ }^{5}$

Acute appendicitis is associated with raised white cell count. It is also raised in many infective conditions thus making it difficult for diagnosis in acute appendicitis. So there is need for scoring system with good sensitivity and diagnostic accuracy to overcome these problems. ${ }^{6}$

There have been various other scoring systems developed of which The Alvarado and modified Alvarado scores have been used commonly, but both Alvarado and modified Alvarado scoring system has low diagnostic value when used in Middle Eastern and Asian populations. ${ }^{7}$

Raja Isteri Pengiran Anak Saleha Appendicitis (RIPASA) is a new scoring system that has been developed for diagnosis of acute appendicitis: which includes fourteen parameters, having better sensitivity, specificity and diagnostic accuracy than other scoring systems in south Asian population. ${ }^{8}$ It was developed in the Department of Surgery at Raja Isteri Pengiran Anak Saleha Hospital, Brunei Darussalam, in 2008 to help in the diagnosis of appendicitis in rural areas and primary health care centres where radiographic imaging systems are not accessible always whereas the patient condition may need for a diagnosis and treatment. The parameters on this scale are mostly based on signs and symptoms, diagnosis and treatment can be done more efficiently and in a more time saving manner also since it's a noninvasive, safe diagnostic method which will guide the surgeon for the management.

The objective of this study is to compare between RIPASA and modified Alvarado scoring system that is suitable for the local population. ${ }^{3}$

\section{METHODS}

This was an observational study conducted among 70 patients presenting with right iliac fossa pain in the department of general surgery at Sri Siddhartha Medical College and Research Institute, Tumkur, between October 2018 and March 2020 for a period of 18 months. A purposive sampling method was used.

\section{Sample Size Calculation}

$n_{s p}=\frac{Z_{\propto / 2}^{2}\left[S_{p}\left(1-S_{p}\right)\right]}{d^{2}(1-\operatorname{Pr} \text { evalence })}$

$Z_{\propto / 2}^{2}=1.96$

$\mathrm{S}_{\mathrm{p}}=0.80$ (specificity of Modified Alvarado scoring)

$\mathrm{d}=0.10$ (margin of error)

$\mathrm{p}=0.14$ (prevalence)

A total of 70 patients presenting with right iliac fossa pain were recruited for this study.

\section{Inclusion Criteria}

All patients $>18$ years of age, presenting with right lower quadrant pain and the patients who underwent appendectomy.

\section{Exclusion Criteria}

1. Patients those who have been admitted by other specialties for other complaints but who subsequently developed right iliac fossa pain.

2. Patients who have received antibiotic in the last 2 days for other complaints.

3. Patient not willing for surgery.

4. Patient with appendicitis in pregnancy.

5. Patient with appendicular mass or abscess.

\section{Method of Collection of Data}

1. Relevant history including age, sex, religion, occupation, nationality, right iliac fossa pain, anorexia, nausea vomiting, and duration of symptom were taken.

2. Relevant examination including right lower quadrant tenderness, right lower quadrant guarding, rebound tenderness, Rovsing sign and fever was done.

3. Relevant laboratory investigations including complete blood count, urine routine, ultrasonography (USG) abdomen and pelvis / contrast-enhanced computed tomography (CECT) abdomen were done.

4. Modified Alvarado and RIPASA scoring were computed as per guidelines.

\begin{tabular}{|cc|}
\hline Signs and Symptoms & Score \\
Migration of pain to right lower quadrant & 01 \\
Anorexia & 01 \\
Nausea and vomiting & 01 \\
Right lower quadrant tenderness & 02 \\
Rebound tenderness & 01 \\
Fever & 01 \\
Leukocytosis & 02 \\
Shift of leucocytes to the left & 01 \\
Total & $\mathbf{1 0}$ \\
\hline Table 1. Modified Alvarado Score $^{\mathbf{8}}$ \\
\hline
\end{tabular}

Institutional ethical forum approved the study. Written consent was obtained for all patients in the study population. The risks and benefits involved in the study were explained to the participants before obtaining consent. Confidentiality of the study participants was maintained.

Modified Alvarado score of $<7$ and RIPASA score of $<7.5$ was taken as cut off to label a patient as high or low possibility of acute appendicitis. Intra operative findings and histopathological examination was correlated with the modified Alvarado and RIPASA score. 
Interpretation of Modified Alvarado Score

1. Score $<5=$ Very unlikely of acute appendicitis

2. Score $5-7=$ May be, acute appendicitis

3. Score $>7=$ Acute appendicitis is probable / definite, operate.

\begin{tabular}{|c|c|}
\hline Patient Characteristics & Score \\
\hline \multicolumn{2}{|l|}{ Gender } \\
\hline Male & 0.5 \\
\hline Female & 1.0 \\
\hline \multicolumn{2}{|l|}{ Age } \\
\hline$<40$ & 1.0 \\
\hline$>40$ & 0.5 \\
\hline \multicolumn{2}{|l|}{ Symptoms } \\
\hline Pain in right iliac fossa & 0.5 \\
\hline Migration of pain to right lower quadrant & 0.5 \\
\hline Anorexia & 1.0 \\
\hline Nausea and vomiting & 1.0 \\
\hline \multicolumn{2}{|l|}{ Duration of Symptoms } \\
\hline Less than 48 hours & 1.0 \\
\hline More than 48 hours & 0.5 \\
\hline \multicolumn{2}{|l|}{ Signs } \\
\hline Right lower quadrant tenderness & 1.0 \\
\hline Guarding & 2.0 \\
\hline Rebound tenderness & 1.0 \\
\hline Rovsing sign & 2.0 \\
\hline Elevated temperature $>37 \mathrm{c}>39 \mathrm{c}$ & 1.0 \\
\hline \multicolumn{2}{|l|}{ Investigation } \\
\hline Increased total leucocyte count & 1.0 \\
\hline Negative urinalysis & 1.0 \\
\hline Total & 16.5 \\
\hline \multicolumn{2}{|c|}{ Table 2. RIPASA Score ${ }^{8}$} \\
\hline
\end{tabular}

\section{Interpretation of RIPASA Score}

1. Score $<5=$ Unlikely of acute appendicitis.

2. Score $5.0-7.5=$ Low probability of acute appendicitis.

3. Score $>7.5=$ Probable $/$ definite acute appendicitis, operate.

Minimal score is 2, maximal total score is 16.5 . The receiver operating curve (ROC) at the optimal cut off threshold score for the new appendicitis scoring system was derived and 7.5 was the cut off.

\section{Statistical Analysis}

The data was computed into an excel sheet. Histopathological examination was considered as primary outcome variable. The sensitivity, specificity, positive predictive and negative predictive values of the scoring system was estimated by comparing the threshold level of score with surgical findings and histopathology findings. P value $<0.05$ was considered statistically significant. Receiver operating curve (ROC) was used for delineating the threshold score levels. The International Business Machines Statistical Package for the Social Sciences (IBM SPSS) version 22 was used for statistical analysis.

\section{RESULTS}

Out of 70 patients, 28 patients were between age group 18 - 24 years, 23 between 25 - 32 years, 9 between $33-40$ years, 10 above 40 years. Pain in abdomen was present in 70 (100\%) patients. Nausea and vomiting in 34 (48.57\%), anorexia in 41 (58.57\%) patients, fever in 26 (37.14 \%) patients, right iliac fossa (RIF) tenderness in $66(94.28 \%)$ patients, rebound tenderness in $59(84.28 \%)$ patients, guarding in $54(77.14 \%)$ patients and Rovsing sign in $46(65.71 \%)$ patients.

\begin{tabular}{|cc|}
\hline Age Group & No. of Patients in Particular Age Group \\
$18-24$ & $28(40 \%)$ \\
$25-32$ & $23(32.8 \%)$ \\
$33-40$ & $9(12.8 \%)$ \\
$>40$ & $10(14.2 \%)$ \\
\hline & Table 3. Age Group \\
\hline
\end{tabular}

\begin{tabular}{|cc|}
\hline Signs and Symptoms & No. of Patients \\
Right lower quadrant pain & $70(100 \%)$ \\
Nausea and vomiting & $34(48.57 \%)$ \\
Anorexia & $41(58.57 \%)$ \\
Elevated temperature & $26(37.14 \%)$ \\
RIF tenderness & $66(94.28 \%)$ \\
Rebound tenderness & $59(84.28 \%)$ \\
Guarding & $54(77.14 \%)$ \\
Rovsing sign & $46(65.71 \%)$ \\
\hline Table 4. Signs and Symptoms \\
\hline
\end{tabular}

Predictive Validity of the Scoring Systems

\begin{tabular}{|ccc|}
\hline Parameter & RIPASA Score & Modified Alvarado Score \\
Sensitivity & $94.1 \%$ & $30.9 \%$ \\
Specificity & $33.3 \%$ & $6.0 \%$ \\
Positive predictive value & $97 \%$ & $100 \%$ \\
Negative predictive value & $80 \%$ & $94.0 \%$ \\
Diagnostic accuracy & $94.26 \%$ & $30.95 \%$ \\
False positive rate & $66.7 \%$ & $94.0 \%$ \\
False negative rate & $5.9 \%$ & $69.1 \%$ \\
\hline \multicolumn{2}{|c|}{ Table 5. Predictive Validity of Modified Alvarado } \\
and RIPASA Score as Compared to HPE $(\boldsymbol{n}=\mathbf{7 0})$ \\
\hline
\end{tabular}

In our study, males were 37 patients $(57.2 \%)$ and females were 33 patients (42.8\%). Out of 70 patients, all were tested positive for acute appendicitis in histopathology. At optimal cut-off threshold of $>7.5$, RIPASA was able to identify 70 appendicitis out of 70 cases in which 70 were positive for appendicitis in histopathology report. RIPASA score showed sensitivity of $94.1 \%$, specificity of $33.3 \%$, positive predictive value of $97 \%$, negative predictive value of $80 \%$ and diagnostic accuracy of $94.26 \%$. At optimal cut-off of $>7$, Modified Alvarado was able to identify 55 appendicitis out of 70 cases in which 55 were positive of appendicitis in histopathology report.

Modified Alvarado score showed sensitivity of $30.9 \%$, specificity of $6.0 \%$, positive predictive value of $100 \%$ and negative predictive value of $94 \%$ and diagnostic accuracy of $30.95 \%$.

The study period was 18 months with total 70 patients. Age group being range from 18 to 76 years. Peak age group was ranging between 18 to 24 years of age ( $40 \%$ ). Males were commonly affected than females with M: F 1.1:1.

Pain abdomen was the most common presentation found in $100 \%$ of patients followed by right iliac fossa tenderness $94.28 \%$ and rebound tenderness $84.28 \%$.

In $64 \%$ of the patients, white blood cell count was found to be elevated more than 10,000 cells / $\mathrm{mm}^{3}$ In Histopathology examination, all $100 \%$ patient were found positive for acute appendicitis. Emergency appendectomy was performed in about $63(90 \%)$ and in 7 (10\%) patients, elective appendectomy was performed. In our study patients, mean hospital stay was 4.05 days. In our study, out of 70 patients, 25 patients showed modified Alvarado score $<7$ and 55 patients $>7$. Whereas, in RIPASA score, 5 patients showed score $<7.5$ and 65 had $>7.5$. 


\section{DISCUSSION}

Acute appendicitis is one of the most common surgical emergencies, the evaluation of which is mainly based on history and clinical findings and they are the most important parameters in arriving to a diagnosis of acute appendicitis. In spite of all this, making a fast and exact diagnosis of acute appendicitis can be tough. Delayed or wrong diagnosis of acute appendicitis can lead to complications of the impending sepsis and infection, leading to perforation, peritonitis, intraabdominal collection and septicemia, with rise in morbidity and mortality of patients. ${ }^{9}$

The present study included 70 cases of acute appendicitis, with age group of patients taken above 18 years. There were 37 males and 33 females in our study. The proportion of males was $52.85 \%$ higher than females $47.14 \%$. The average age of the study population was 31.2 years and that was higher when compared to similar study by Singh SK et al. where the mean age was 29.64 years. ${ }^{10}$

In this study, the patients with right lower quadrant pain were $100 \%$ which is similar to study conducted by Srikantaiah $\mathrm{H}$ et al. and Naresh et al. which showed all $100 \%$ participants had right iliac fossa pain. ${ }^{11,12,}$

In the present study, $58.57 \%$ of the patients had anorexia. The patients with nausea and vomiting were $48.57 \%$ and $37.14 \%$ of the patients had fever. In Naresh G et al. a total of 34 patients (34\%) had anorexia while $47 \%$ patients had nausea and vomiting and $97.4 \%$ had fever. ${ }^{12}$

In our study, $94.28 \%$ of the patients had right iliac fossa tenderness. $84.28 \%$ of patients had shown rebound tenderness. $77.14 \%$ of the patients had guarding and Rovsing sign was seen in $65.71 \%$ of cases. In Naresh G et al. study all the patients had right iliac fossa tenderness. The percentage of guarding, rebound tenderness and Rovsing sign among patients was $22 \%, 58 \%$ and $29 \%$ respectively. ${ }^{12}$

In our study, $100 \%$ of the patients showed positive results in ultrasonography and $95.71 \%$ showed positive histopathology report.

Modified Alvarado score when applied to the study population, had 53 cases with a total of $\geq 7$ and 17 with total of less than 7 . On evaluating with respect to the histopathology, the sensitivity, specificity, positive predictive value and negative predictive value of the scoring system in the present study came to be $30.9 \%, 6.0 \%, 100 \%$ and $94.0 \%$ respectively.

RIPASA score when applied to the study population, had 66 patients in $>=7.5$ group and 4 cases with a score of less than 7.5 score group. On evaluating with respect to the histopathology, the sensitivity, specificity, positive predictive value and negative predictive value of the scoring system in the present study came to be $94.1 \%, 33.3 \%, 97.0 \%$ and 80.0 $\%$ respectively.

The findings from the present study matched with the Chong CF et al. study. In this study, sensitivity of RIPASA score is (94.1\%) which is higher when compared with modified Alvarado score (30.9\%). Specificity of the RIPASA score is also more $(33.3 \%)$ when compared with the Modified Alvarado score (6.0\%). RIPASA score also has high diagnostic accuracy of $94.26 \%$ when compared to modified Alvarado score which is $30.95 \% .^{7}$ These results showed to be considered under studies limitations. There is a need for further studies to demonstrate the utility of RIPASA scoring system in variable settings.

\section{CONCLUSIONS}

RIPASA scoring is an easy and reliable scoring system and has better diagnostic accuracy compared to modified Alvarado scoring. It can be introduced in casualty department in primary health care centres as it is a reliable scoring system which is used for quick transfer of patients for immediate surgery and to prevent risk of converting into complicated appendicitis. It does not make use of any higher level investigations and it is completely based on clinical features and simple laboratory investigations. Use of the scoring systems promotes the diagnostic accuracy and subsequently reduces the negative appendectomy rates. As the RIPASA score has high sensitivity and specificity as compared to modified Alvarado scoring system, RIPASA score can be a useful tool in making clinical decisions. Due to the advancement of imaging modalities, these scoring systems have less significant values in tertiary care centres. However, it can be used in areas which lack imaging modalities like rural areas or in primary health care centres where these scoring systems can be used to plan the management of the patients. Thus, RIPASA scoring system is easy, simple, cheap, noninvasive, safe, fast, reliable for pre-operative prediction of acute appendicitis.

\section{Limitations}

This study was only an observational study. Hence the observed association cannot be interpreted as casual inferences. Non-probability sampling technique was employed for the study which is not exact depiction of common population.

\section{Recommendations}

There is a need for further large-scale studies on the subject to further demonstrate the utility of scoring systems.

Data sharing statement provided by the authors is available with the full text of this article at jemds.com.

Financial or other competing interests: None.

Disclosure forms provided by the authors are available with the full text of this article at jemds.com.

\section{REFERENCES}

[1] Barman MK, Das K, Mukherjee K. A study on comparison of diagnostic efficiency between Modified Alvarado Score and graded compression Ultrasonography in the case of acute appendicitis. Int J Curr Res Rev 2018;10(9):22-6.

[2] Williams NS, Bulstrode CJK, O'Connell PR. The vermiform appendix. In: Bailey and Love Short Practice of Surgery. 26 ${ }^{\text {th }}$ edn. CRC Press 2013: p. 1205.

[3] Gilmore OJ, Browett JP, Griffin PH, et al. Appendicitis and mimicking conditions. A prospective study. Lancet 1975;2(7932):421-4. 


\section{Jemds.com}

[4] Alvarado A. A practical score for the early diagnosis of acute appendicitis. Ann Emerg Med 1986;15(5):557-64.

[5] Fitz RH. Perforating inflammation of the vermiform appendix: with special reference to its early diagnosis and treatment. Am J Med Sci 1886;92(184):32-46.

[6] Lau WY, Ho YC, Chu KW, et al. Leukocyte count and neutrophil percentage in appendicectomy for suspected appendicitis. Aust N Z J Surg 1989;59(5):395-8.

[7] Chong CF, Adi MI, Thien A. Development of the RIPASA score: a new appendicitis scoring system for the diagnosis of acute appendicitis. Singapore Med J 2010;51(3):220-5.

[8] Chong CF, Thien A, Mackie AJA. Comparison of RIPASA and alvarado scores for the diagnosis of acute appendicitis. Singapore Med J 2011;52(5):340-5.

\section{Original Research Article}

[9] Tatar IG, Yilmaz KB, Sahin A, et al. Evaluation of clinical alvarado scoring system and CT criteria in the diagnosis of acute appendicitis. Radiol Res Pract 2016;2016:9739385.

[10] Singh SK, Singh KK, Singh C, et al. Comparative study of diagnostic accuracy of modified alvarado score and ultrasonography in acute appendicitis. IOSR Journal of Dental and Medical Sciences 2014;13(1):36-40.

[11] Srikantaiah HC, Arvind NK. Validation of RIPASA scoring system for the diagnosis of acute appendicitis. Journal of Evolution of Medical and Dental Sciences 2015;4(100):16533-48.

[12] Naresh G, Reddy MV, Inamdar P. Evaluation of acute appendicitis according to RIPASA scoring system. Indian J Appl Res 2018;8(9). 